Military Technical College

Kobry El-Kobbah, Cairo, Egypt

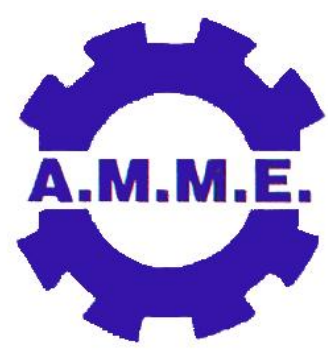

$14^{\text {th }}$ international Conference On Applied Mechanics And Mechanical Engineering Amme-14

\title{
Modeling and Simulation of Tracked Vehicles Suspension
}

\author{
By
}

A.M. Salem*

\section{Abstract:}

Preserving drive smoothness is one of the basic requirements imposed on tracked vehicles operation. Drive smoothness that depends on design and quality of vehicle suspension is accomplished by minimum vibration of vehicle hull during drive. The excessive vibration arises particularly at high vehicle speed can harm the operation of vehicle crew and stability of vehicle equipment. In the present paper, a full vehicle model is developed with the objective of modeling suspension system dynamic response. The vehicle model involving few degrees of freedom is applied for Armored Personnel Carrier M113 where is Lagrangian principle is used. A computer simulating model for the vehicle suspension is developed in order to evaluate the response of vehicle hull oscillation due to different excitations resulting from movement over irregular roads and due to different vehicle speed. A parametric investigation was carried out using the validated model in order to study the effect of suspension parameters, like suspension stiffness and shock absorber damping coefficient, on vehicle vibrational performance.

\section{Keywords:}

Tracked vehicle, suspension, dynamics, shock absorber, torsion bar

\footnotetext{
${ }^{*}$ Egyptian Armed Forces
} 


\section{Introduction}

Oscillation of high mobility tracked vehicles hull has a bad effect on the accuracy of fire on move, observation of roadway and battlefield by means of observation instruments, crew fatigue, reliability of vehicle systems, and their capability as combat vehicles. Therefore it is of primary importance for modern military tracked vehicles to improve drive smoothness over rough terrain surfaces. The maximum allowable vehicle speed varies with the roughness of a particular terrain, and is primarily influenced by the suspension system design.

Tracked vehicles are differing from wheeled vehicle by their undercarriage. Tracked vehicles undercarriage comprises two major groups, track moving system and suspension system. The suspension system is the group of all components connecting the vehicle road wheels with hull. It includes elastic elements, shock absorbers, axle arms and road wheels stroke limiters. Due to the operation of tracked vehicles, as combat vehicles, on different roads, they are exposed to different excitations and hence various vibrations. The suspension system is responsible for softening and attenuation of vehicle vibration while moving on such roads. The amplitude and frequency of vibration depend on the design and quality of vehicle suspension. In this paper, evaluation of tracked vehicle suspension characteristics and vibrational response as a function of suspension elements parameters is studied [1-4].

The vibrational response of tracked vehicle depends mainly on the characteristics of suspension elements which must be identified separately and introduced in the equation of motion of vehicle hull to obtain its response. Evaluation of APC M113 vibrational response, due to different excitations, has been carried out analytically using Lagrange's principle in order to study the influence of suspension element parameters.

A computer simulation model is carried out to analyze and improve the expected field performance of tracked vehicle suspension. It is realized using the Automotive Dynamic Analysis of Mechanical System (ADAMS). The dynamic program is used to simulate the vehicle movement over different road profiles with different speeds.

\section{Suspension System Parameters}

The most effective parameters in tracked vehicles suspension are the suspension stiffness and shock absorber damping coefficient. Evaluation of these parameters is essential when looking for the vibration response of vehicle. Hereafter, identification of the characteristics of suspension parameters will be carried out.

\section{Suspension characteristics}

Torsion bar characteristics are similar to spring characteristics as the torsion bar performs spring action. The torsion bar stiffness is expressed by the ratio of the deforming torque and the corresponding twist angle measured in the direction of the moment. Suspension characteristics represent the relationship between the vertical force $P_{w}$ acting on the road wheel and the corresponding vertical displacement $f_{w}$. Figure (1) demonstrates the suspension characteristics of APC M113 [5]. It can be seen from the figure that this characteristic is quasi linear and the suspension stiffness $C_{i}$ has an average value of 133 $(\mathrm{kN} / \mathrm{m})$. 


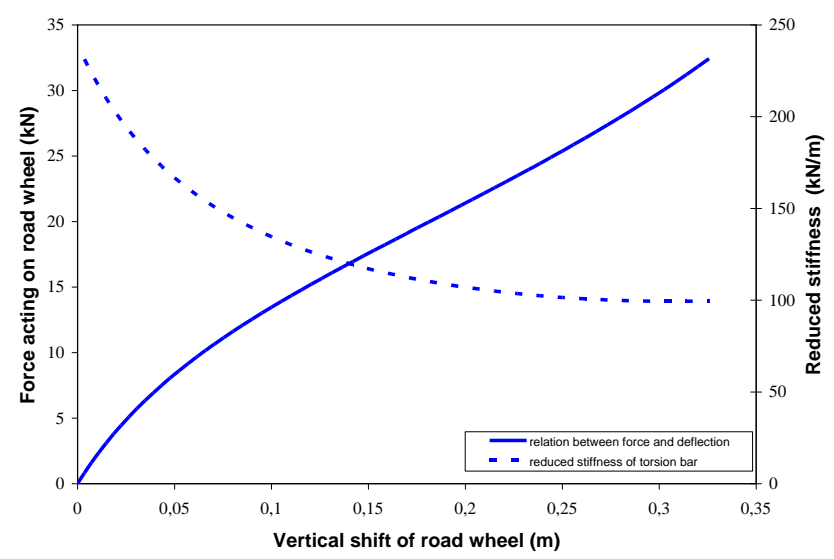

Figure (1): Suspension characteristics of APC M113

\section{Shock absorber damping coefficient}

The damping coefficient of shock absorber is determined from the condition of the required degree of decrease of amplitudes of oscillations in a certain period of time. Knowing the period $T_{d}$ in which the amplitude of oscillation is required to decrease from $\varphi_{1}$ to $\varphi_{2}$, as shown in

Figure (2), The average value of damping coefficient can be calculated according to the following formula [6]:

$$
\mu=\frac{\ln \delta}{\sum_{1}^{k d} l_{i}^{2}} \frac{J_{y} \omega_{\varphi}}{\sqrt{4 \pi^{2}+(\ln \delta)^{2}}}
$$

where $\delta$ is the degree of decrease of amplitude of oscillations $\left(\delta=\frac{\varphi_{1}}{\varphi_{2}}\right), \omega_{\varphi}$ is the natural frequency of angular oscillation of vehicle around its lateral axis, $J_{y}$ is the moment of inertia of the vehicle about this axis, $k_{d}$ is the number of shock absorber in one side of vehicle, and $\mathrm{l}_{\mathrm{i}}$ is the distance of absorbers from vehicle center of gravity.

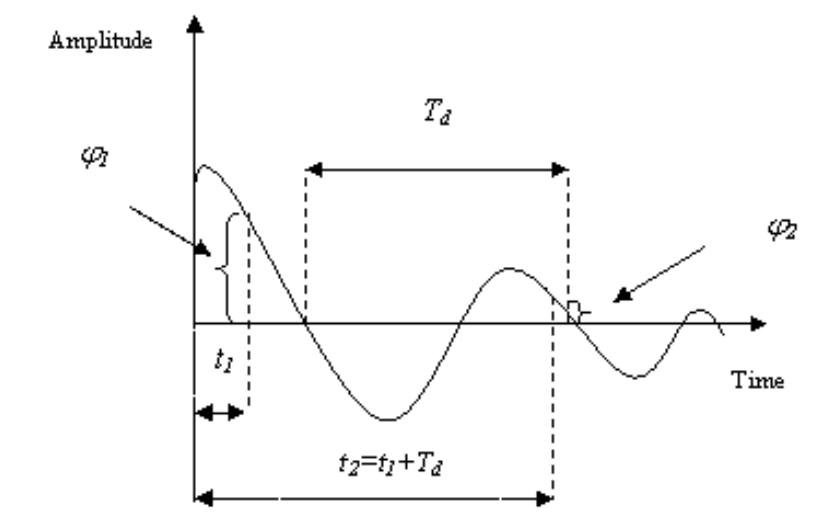

Figure (2): Illustration of two successive amplitudes

In tracked vehicles, the damping coefficient of shock absorbers has a different value in both rebound and compression strokes. Figure (3) demonstrates the variation of the applied force on the shock absorber of APC M113 with velocity at low excitation frequencies while table 1 shows the values of damping coefficient in compression and rebound strokes [7] 


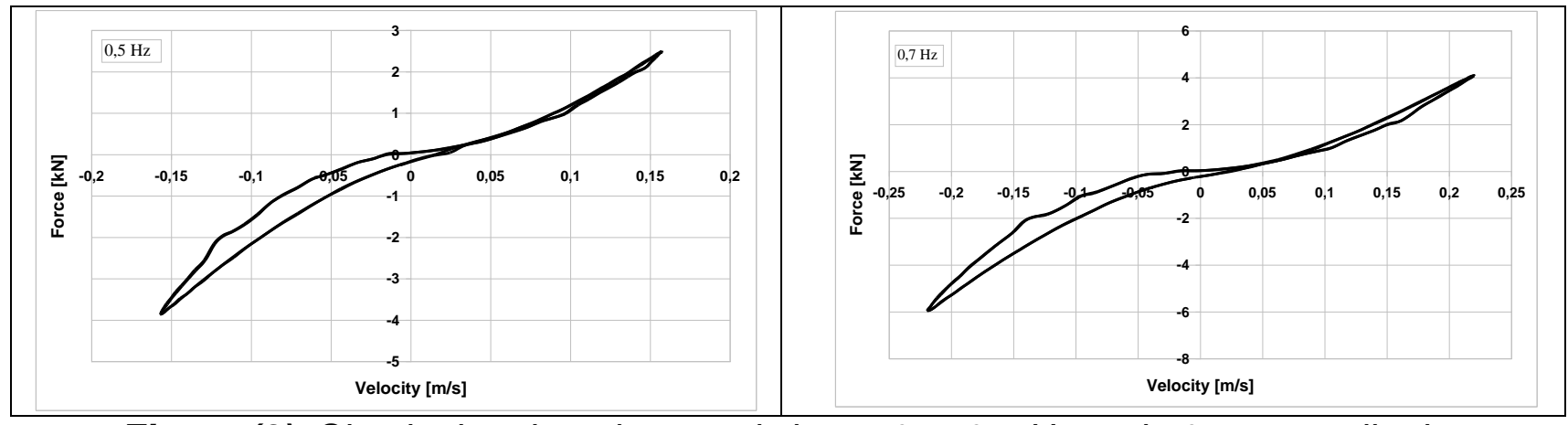

Figure (3): Shock absorber characteristics at 0.5, $0.7 \mathrm{~Hz}$ and $50 \mathrm{~mm}$ amplitude

Table (1): Average value of damping coefficient of APC M113

\begin{tabular}{|c|c|c|c|}
\hline \multirow{2}{*}{ Frequency $(\mathrm{Hz})$} & \multicolumn{3}{|c|}{ Damping coefficient $(\mathrm{kN} . \mathrm{s} / \mathrm{m})$} \\
\cline { 2 - 4 } & compression $\left(\mu_{1}\right)$ & rebound $\left(\mu_{2}\right)$ & average value $(\mu)$ \\
\hline 0.5 & 11.9 & 19.8 & 15.9 \\
\hline 0.7 & 13.6 & 20.9 & 17.2 \\
\hline
\end{tabular}

\section{Differential Equations of Vehicle Hull Oscillation}

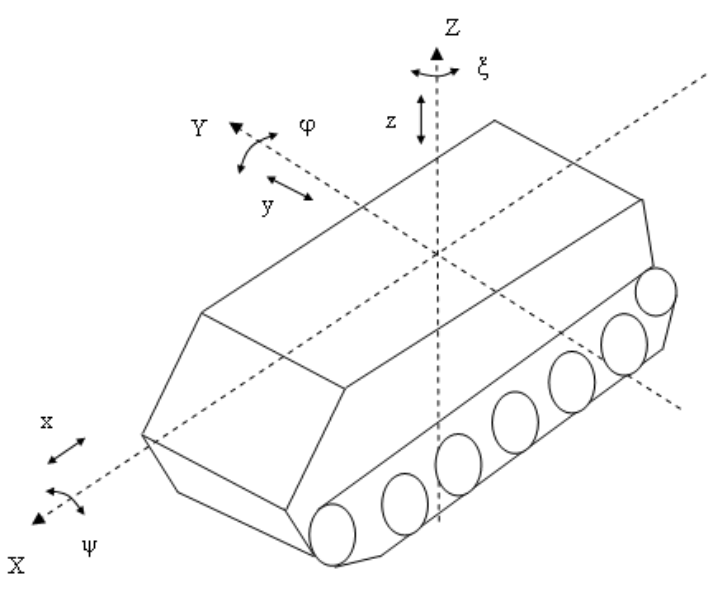

Figure (4): Types of vehicle hull oscillation

The vehicle is considered as multi-degree of freedom system and it can be modeled by a mass $m$ suspended on a number of springs each with stiffness $C$ and dampers with average damping coefficient $\mu$. When the vehicle is derived over unevenness the elastic elements are deformed resulting in hull vibration. The vehicle hull in general as a vibrating system has six degrees of freedom (rotation about $\mathrm{x}, \mathrm{y}$ and $\mathrm{z}$ by angles $\psi, \varphi$, and $\xi$ respectively and translation along such axes) as shown in Figure (4). As practically the vibration of $x, y, \zeta$ are neglected due to their low effect, so the following types of vibration will be only concerned:

- Vertical vibration with linear displacement $z$

- Longitudinal angular vibration with angular displacement $\varphi$

- Lateral angular vibration with angular displacement $\psi$

In the analysis of such types of vibrations, the following assumptions are considered:

- the road wheels are identically arranged on both sides of vehicle

- the elastic elements are of linear characteristics (constant suspension stiffness)

- the tires of road wheels are of infinite rigidity

- null friction in the suspension 


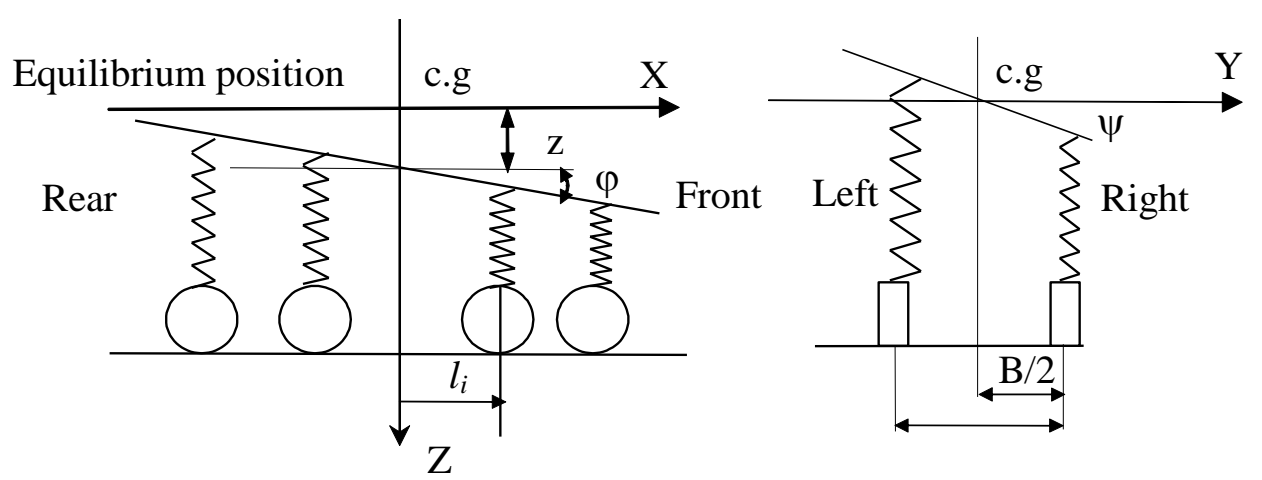

Figure (5): Coordinates system

The differential equations of hull oscillation are deduced using Lagrange equation:

$$
\frac{d}{d t}\left(\frac{\partial T}{\partial \dot{q}_{i}}\right)-\frac{\partial T}{\partial q_{i}}=-\frac{\partial U}{\partial q_{i}}
$$

Where $T$ and $U$ are kinetic and potential energies of vehicle suspended mass $m_{s}$ respectively and $q_{i}$ is the generalized coordinates.

$$
\begin{gathered}
T=\frac{1}{2}\left(m_{s} \dot{z}^{2}+J_{y} \dot{\varphi}^{2}+J_{x} \dot{\psi}^{2}\right) \\
U=\frac{1}{2} \sum_{1}^{k} C_{i} f_{i R}^{2}+\frac{1}{2} \sum_{1}^{k} C_{i} f_{i L}^{2}
\end{gathered}
$$

Where $f_{i R, L}$ is the deflection of reduced springs of the right and left hand sides of the vehicle respectively and $k$ is the number of torsion bars on one side of the vehicle. By substituting $T$ and $U$ into Lagrangian equation we obtain a system of differential equations expressing the natural oscillations of the vehicle hull as follows.

$$
\begin{gathered}
m_{s} \ddot{z}+z \sum_{1}^{k} 2 C_{i}+\varphi \sum_{k 2}^{k 1} 2 C_{i} l_{i}=0 \\
J_{y} \ddot{\varphi}+\varphi \sum_{1}^{k} 2 C_{i} l_{I}^{2}+Z \sum 2 C_{i} l_{i}=0 \\
J_{x} \ddot{\psi}+\psi \sum_{1}^{k} 2 C_{i} \frac{B^{2}}{2}=0
\end{gathered}
$$

The Lateral angular oscillation $\psi$ occurs less frequently compared to the oscillations $\varphi$ and z. Even if it is occurred it will be damped by the side shift of road wheels on tracks or by the tracks on the ground. Consequently, the above equations can be rearranged in a simple form after neglecting the lateral vibration as:

$$
\begin{gathered}
\ddot{z}+a z+b \varphi=0 \\
\ddot{\varphi}+\frac{e}{\rho_{y}^{2}} \varphi+\frac{b}{\rho_{y}^{2}} z=0
\end{gathered}
$$

where $\mathrm{a}, \mathrm{b}, \frac{e}{\rho_{y}^{2}}$ are the functions of constructional parameters: 


$$
a=\frac{1}{m_{s}} \sum_{1}^{k} 2 c_{i}, \quad b=\frac{1}{m_{s}} \sum_{k_{2}}^{k} 2 c_{i} l_{i}, \quad e=\frac{1}{m_{s}} \sum_{1}^{k} 2 c_{i} l_{i}^{2}, \quad \rho_{y}^{2}=\frac{J_{y}}{m_{s}}
$$

It is found that the coordinates $z$ and $\varphi$ are coupled together with the coefficient $\mathrm{b}$, which means that if a vertical force is applied at the vehicle center of gravity, the hull will undergo a linear displacement $z$ and a rotation $\varphi$ as well. On the other hand, the moment acting on the hull will cause its rotation by an angle $\varphi$ and a linear displacement $z$. For the case where the suspension system is symmetric about the vehicle vertical axis, the quantity $\sum_{1}^{k 1} 2 C_{i} l_{i}$ for the front road wheels is equal to that of the rear so that the coupling coefficient $b$ is equal to zero. Accordingly, the equations of motion can be expressed as follows:

$$
\begin{gathered}
\ddot{z}+a z=0 \\
\ddot{\varphi}+\frac{e}{\rho_{y}^{2}} \varphi=0
\end{gathered}
$$

\section{Natural frequency and solution of differential equation}

Due to small vertical oscillation with respect to angular oscillation, the angular oscillation $\varphi$ is only considered. The solution of differential equation of angular oscillation has the following form:

$$
\phi=A \cos \left(\omega_{\varphi} t+\alpha\right)
$$

where $\omega_{\varphi}$ is the natural frequency of angular oscillation and $\alpha$ is the phase angle.

$$
\omega_{\varphi}=\sqrt{\frac{e}{\rho_{y}^{2}}}=\sqrt{\frac{\sum_{1}^{k} 2 C_{i} l_{i}^{2}}{J_{y}}}
$$

This indicates that $\omega_{\varphi}$ not only depends on the suspension stiffness $C_{i}$ but also on road wheels distribution $l_{i}$ and hence to decrease $\omega_{\varphi}$, shorter hull beside soft springs is to be used. The complete solution of differential equation is found by applying the initial conditions which leads to:

$$
\varphi=\varphi_{o} \cos \omega_{\varphi} t
$$

Where $\varphi_{0}$ is the initial angular displacement. In tracked vehicles, the maximum value of amplitude of angular oscillation is usually $3^{\circ}-5^{\circ}$. In the following sections, the study of damped free and forced vibrations of vehicle hull will be presented.

\section{Damped free vibration of vehicle hull}

During hull oscillation, there exists a damping force originated from the operation of hydraulic shock absorbers. The shock absorber used in APC M113 is of viscous type where the damping force is proportional to the velocity of road wheel. In this kind of vehicle, the dampers are connected to the first, second, and fifth road wheels on both vehicle sides in order to provide an effective damping moment. In addition to the assumptions mentioned previously the damping is considered linearly dependent on wheel velocity. The moments acting on vehicle hull are indicated in Figure (6). 


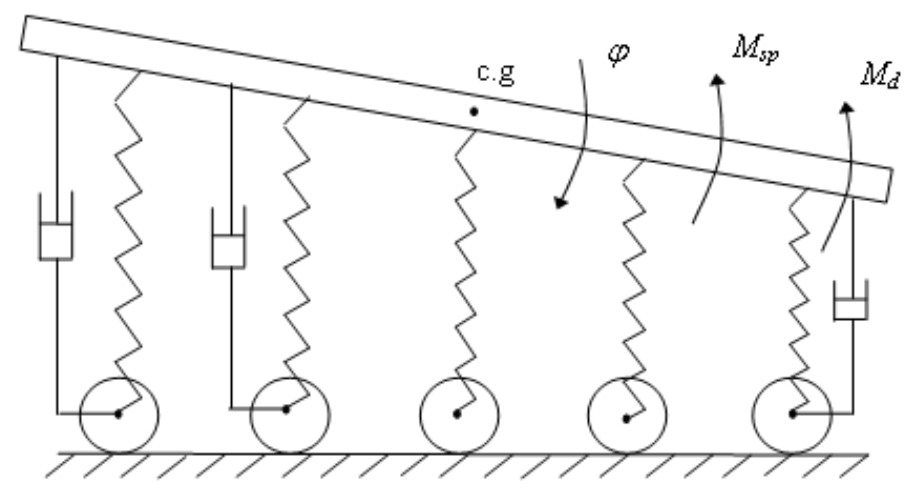

Figure (6): Acting moments on hull in free damped vibration

The equation of motion of hull in this case will have the form:

$$
J_{y} \ddot{\varphi}=-M_{s p}-M_{d}
$$

Where $M_{s p}$ is the moment of springs forces while $M_{d}$ is the moment of damping forces, so that:

$$
M_{s p}=2 \varphi \sum_{1}^{k} C_{i} l_{i}^{2} \text { and } M_{d}=2 \mu \dot{\varphi} \sum_{1}^{k_{d}} l_{i}^{2}
$$

Substituting these relations of $M_{s p}$ and $M_{d}$ in the above differential equation of free damped vibration:

$$
\ddot{\varphi}+2 n \dot{\varphi}+\omega_{\varphi}^{2} \varphi=0
$$

It is a homogenous second order linear differential equation where its solution after applying the initial conditions is:

$$
\varphi=\frac{\varphi_{o}}{\cos \alpha_{o}} e^{-n t} \cos \left(\omega_{d} t+\alpha_{o}\right)
$$

where $\omega_{d}=\sqrt{\omega_{\varphi}^{2}-n^{2}}$ is the frequency of damped oscillation

Figure (7) illustrates the vibrational response of APC M113 in case of free damped vibration. The graph indicates the variation of the angular displacement with time. It can be observed that the amplitude of oscillation is decreased exponentially with time. The degree of decrease of such oscillation depends on the value of damping coefficient of shock absorbers $\mu$ and also on their number and locations.

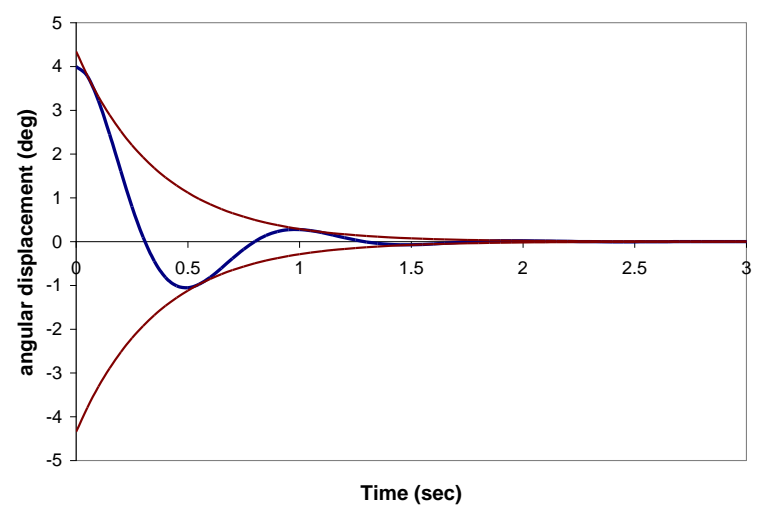

Figure (7): Vehicle response of free damped vibration 


\section{Damped forced vibration of vehicle hull}

During vehicle drive, it passes by road unevenness which acts continuously on vehicle hull through the springs and shock absorbers representing an external excitation causing the hull to undergo forced vibrations. The nature of this vibration, beside the vehicle speed, depends on the shape and dimension of unevenness. The road profile is ultimately changed and it is impossible to simulate exactly this profile. One of the most widely applied road models for tracked vehicles is the model in which the road unevenness is considered to be of sinusoidal shape as shown.

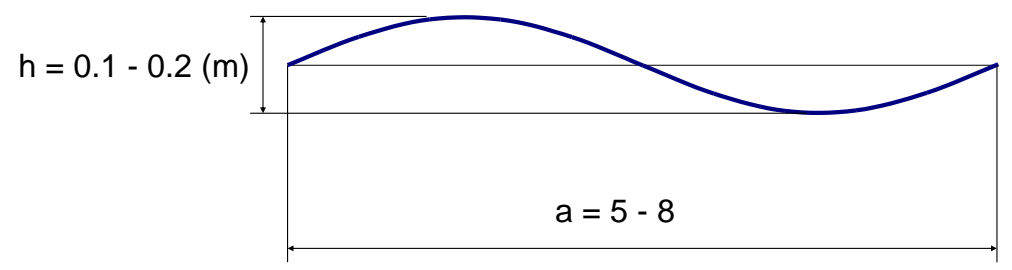

Figure (8): Model of road unevenness

The coordinate $X$ measured from the beginning of road unevenness is considered beside the coordinates $Z$ and $\varphi$ as shown in Figure (9).

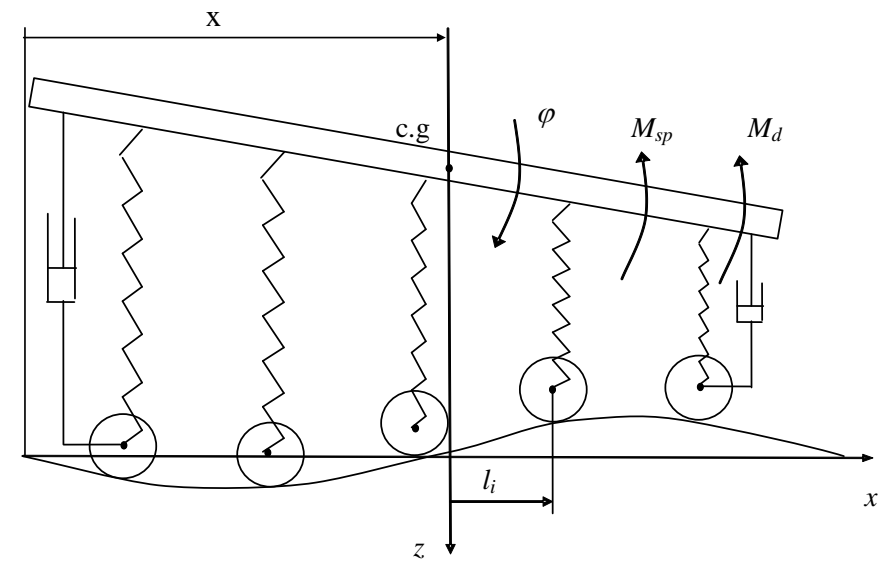

Figure (9): Moments acting on vehicle hull in damped forced vibration

The equation of motion in this case will be:

$$
J \ddot{\varphi}=-M_{s p}-M_{d}
$$

where $M_{s p}$ and $M_{d}$ are the moments of all springs and damping forces so that:

$$
\begin{array}{crl}
M_{s p}=2 \sum_{1}^{k} F_{i} l_{i} & \quad & \mathrm{~F}_{\mathrm{i}}=C_{i} f_{i} \\
M_{d}=2 \sum_{1}^{k_{d}} R_{i} l_{i} & \mathrm{R}_{\mathrm{i}}=\mu \frac{d f_{i}}{d t} l_{i}
\end{array}
$$

The deflection $f_{i}$, represents the total deformation resulting from hull rotation by angle $\varphi$ and vehicle run over the unevenness so that:

$$
f_{i}=\varphi l_{i}-\frac{h}{2} \sin 2 \pi \frac{x+l_{i}}{a}
$$

By substituting expressions of $M_{s p}$ and $M_{d}$ and considering $C_{i}$ to be constant $=C_{w}$, the differential equation of motion will be:

$$
\ddot{\varphi}+2 n \dot{\varphi}+\omega_{\varphi}^{2} \varphi=E \cos q t+D \sin q t
$$


with $q=\frac{2 \pi V}{a} \quad, \quad E=\frac{h C_{w} \sum_{1}^{k} l_{i} \sin \frac{2 \pi l_{i}}{a}}{J_{y}} \quad, \quad D=\frac{-\mu h q \sum_{1}^{k d} l_{i} \sin \frac{2 \pi l_{i}}{a}}{J_{y}}$

The general solution of the above equation will be the sum of general solution of homogenous equation and particular solution of non-homogenous equation, so that:

$$
\varphi=e^{-n t}\left(C_{1} \cos \omega_{d} t+C_{2} \sin \omega_{d} t\right)+M \cos q t+N \sin q t
$$

The first term in solution represents the response of damped free oscillation with frequency $\omega_{d}$ and the second two terms represent the response of forced oscillation with frequency $q$. Constants $M, N, C_{1}$ and $C_{2}$ are determined as follows:

$$
\begin{array}{crr}
M=\frac{E\left(\omega_{\varphi}^{2}-q^{2}\right)-2 n q D}{\left(\omega_{\varphi}^{2}-q^{2}\right)^{2}+4 n^{2} q^{2}} & , & N=\frac{D\left(\omega_{\varphi}^{2}-q^{2}\right)+2 n q E}{\left(\omega_{\varphi}^{2}-q^{2}\right)^{2}+4 n^{2} q^{2}} \\
C_{1}=\varphi_{o}-M & , & C_{2}=\frac{\dot{\varphi}_{o}+n \varphi_{o}-n M-q N}{\omega_{d}}
\end{array}
$$

The response of the vehicle in this case of forced damped vibration is shown in Figure (10). It is shown from the figure that the damped free oscillation disappears rapidly with time and remains only the forced oscillation in the steady state.

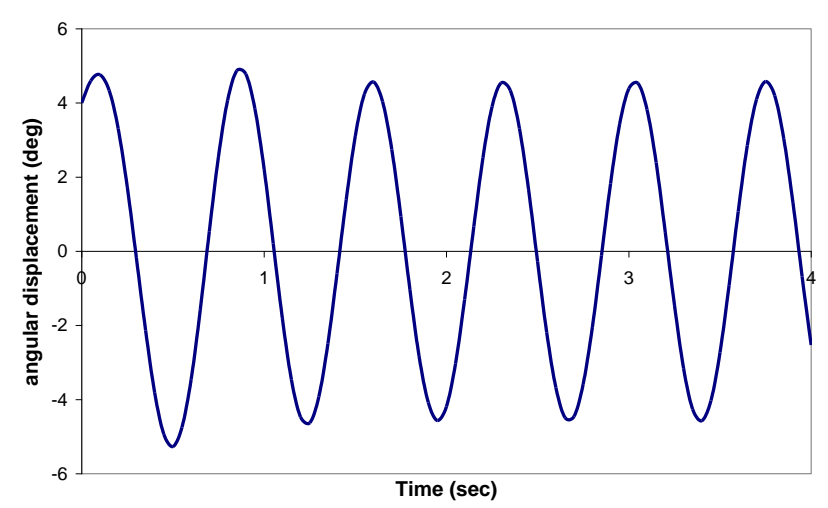

Figure (10): Vehicle response of forced damped oscillation

\section{Representation of Vehicle Suspension Using a Computer Simulated model}

A dynamic simulated model is carried out using the package of Automotive Dynamic Analysis of Mechanical System (ADAMS) to simulate the suspension system of APC M113 and to find the vibrational response of the vehicle due to its drive over irregular terrain. Through ADAMS/View one can build models of mechanical systems and simulate the fullmotion behavior of the vehicle. ADAMS/View can be used also to quickly analyze multiple design variations until finding the optimal design [8-9].

In ADAMS all structural components like body and suspension are modeled as rigid parts. To increase the model accuracy, the assumptions mentioned in the analytical study are excluded, so the model can represent the vibration response of vehicles more accurately than does the analytical one. The road profile is used as an input excitation for the model by imposing on each road wheel a different vertical displacement. Each displacement represents the vertical shift, $f_{i}$, of the road wheel resulting from road irregularity so that:

$$
f_{i}=-\frac{h}{2} \sin 2 \pi \frac{x+l_{i}}{a}
$$


The vehicle in the model is suspended on ten road wheels on both vehicle sides. Each one is connected with the hull through a spring with stiffness equal to suspension stiffness. The first, second and fifth road wheels on each side of the vehicle are equipped with hydraulic shock absorbers. The damping coefficient of each shock absorber is calculated according to the relation mentioned before. The real characteristics of suspension and shock absorbers are introduced in the model using spline functions. Figure (11) represents the full suspension model for APC M113 where it indicates each suspension unit of road wheels.
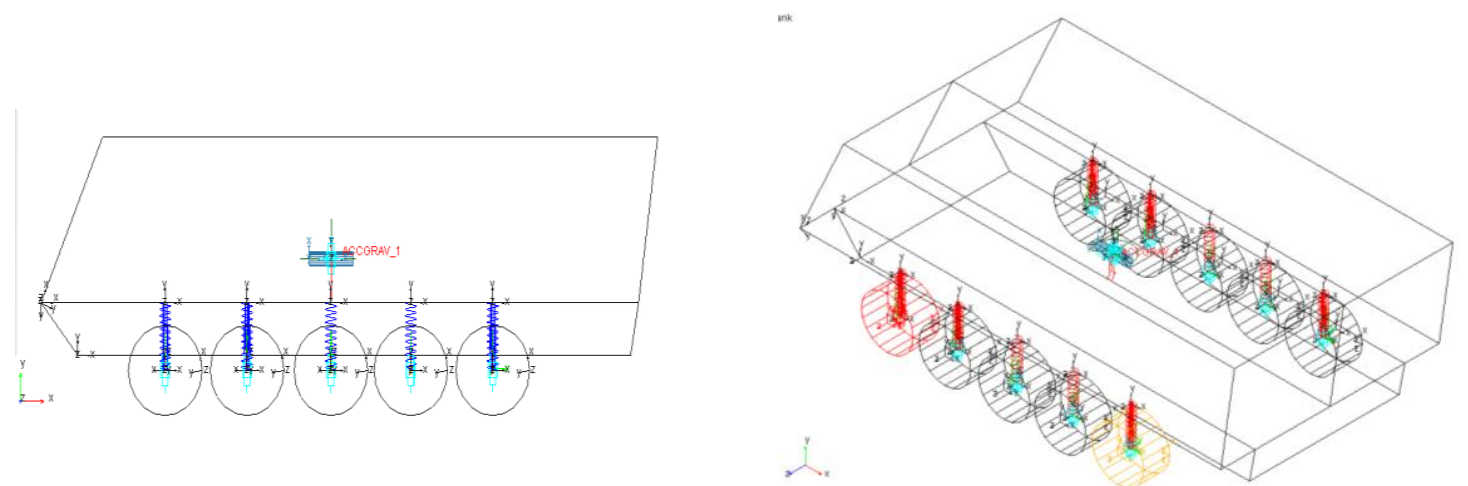

Figure (11): Simulating model of APC M113

\section{Results and Analysis}

Figure 12 compares the results obtained by the simulating model in case of using the real characteristics of suspension stiffness with those obtained by the analytical solution. It can be seen that the model can simulate satisfactory the vibrational response of the vehicle. The little difference shown in the figure is due to the fact that the model simulates the vehicle response with more accurate results than does the analytical representation because of excluding the assumption mentioned in the analytical investigation. Therefore it is more accurate to use the simulating model when modeling the vehicle suspension system and searching for the vibrational response.

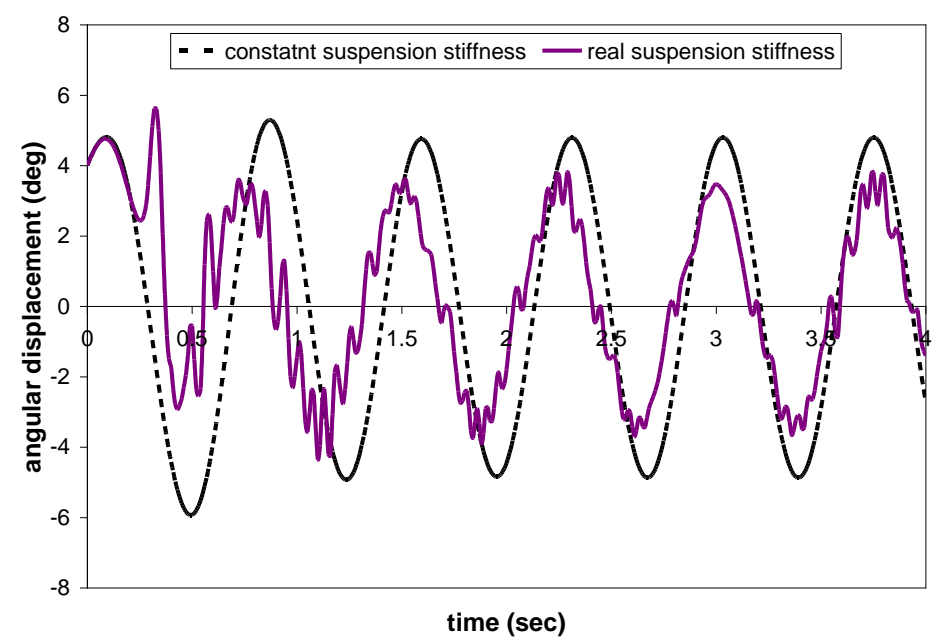

Figure (12): vehicle response with real suspension characteristics

\section{Parametric Study}

A parametric study concerning the effect of suspension parameters and road profile on the vibrational response of the vehicle has been carried out. This study is executed using the simulating model. 


\section{Effect of suspension parameters}

The suspension parameters include suspension stiffness and shock absorber damping coefficient. Figure 13 shows the effect of suspension stiffness on vehicle response while figure 14 shows the effect of shock absorber coefficient on such response.

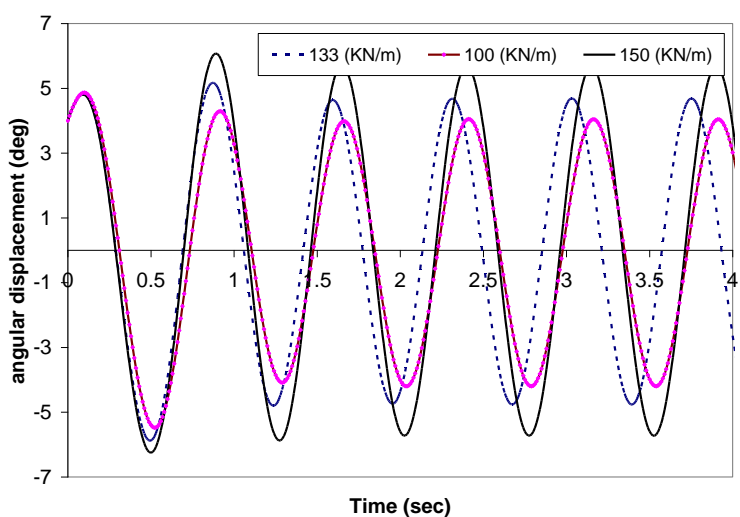

Fig. 13: Effect of suspension stiffness

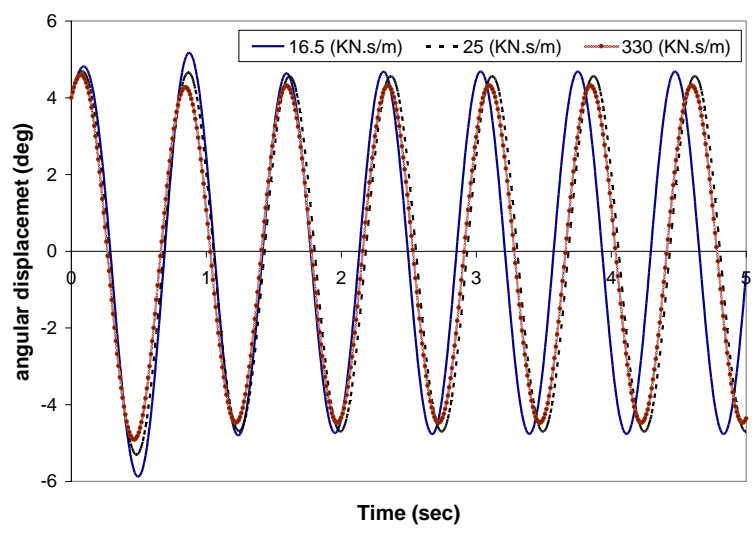

Fig. 14: Effect of damper coefficient

In figure 13, the effect of suspension stiffness shows that the amplitude of vibration is decreased with the decrease of suspension stiffness. Consequently if soft springs are used in the suspension system, small amplitude of vibration will be attained. Figure 14 shows that the amplitude and frequency of vibration decrease with the increase of damping coefficient. The vibration response shown in both figures consists of two types of oscillations: damped free oscillation with damped frequency $\omega_{d}$ which disappears rapidly with time and forced oscillation with excitation frequency q which remains only in the steady state.

\section{Effect of road profile}

The profile of sinusoidal road is changed in frequency and amplitude. Figure 15 and 16 illustrate the effect of road frequency and amplitude on the vibrational response of forced vibration respectively. The frequency of excitation depends on the road frequency and vehicle velocity. It is shown from figure 15 that as the vehicle velocity increases, the amplitude of vibration decreases and the frequency of vibration decreases. It is preferred to change the vehicle velocity for a given road profile such that the resonance frequency is avoided.

As the road amplitude increases, the amplitude of excitation increases and hence the amplitude of vibration of vehicle hull increases as shown in figure 16. The figure depicts the vehicle vibration at different road amplitudes such that the maximum amplitude of vibration is obtained at the higher road amplitude.

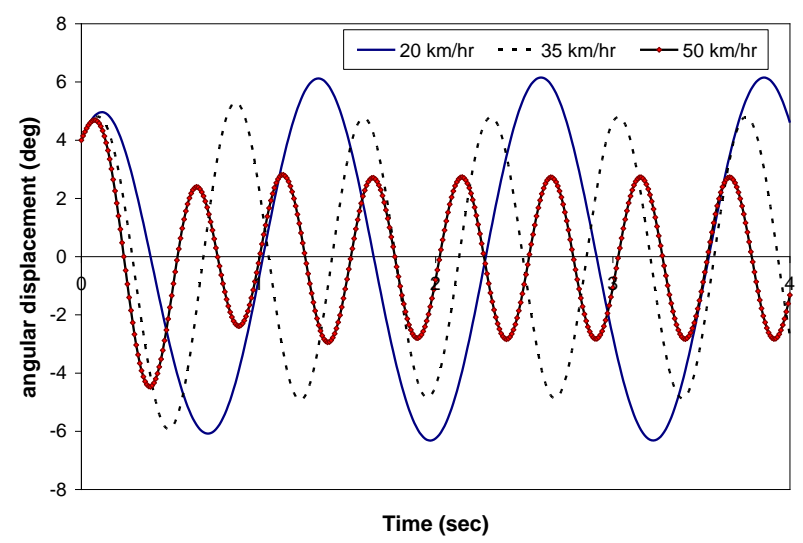

Fig. 15: Effect of excitation frequency

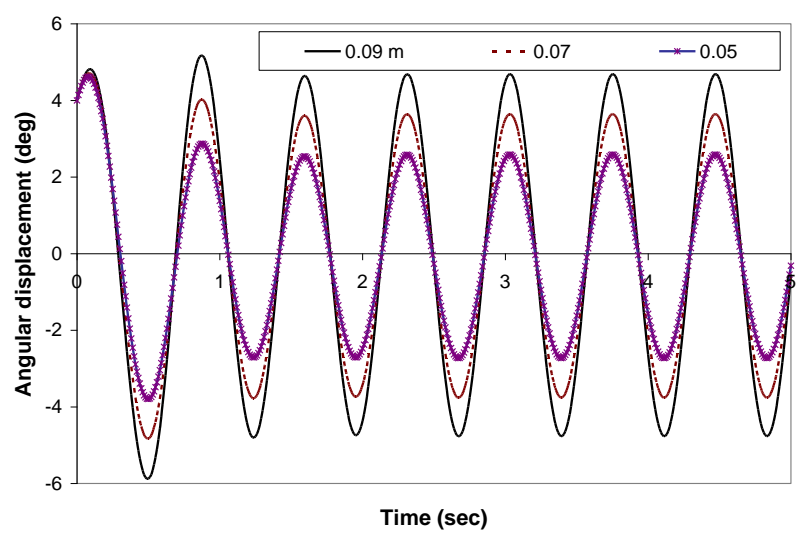

Fig. 16: Effect of excitation amplitude 
From the previous parametric study, it is concluded that each parameter has its own effect and it is difficult to identify a constant value for each one. Therefore it is better to change the parameters of suspension according to road excitation. This fact results in studying the possibility of integrating the active suspension with tracked vehicles.

\section{Conclusions}

- In this study an improved simulating model is developed for the APC M113 suspension system. This model provides the vibrational response of the vehicle in dependent with different parameters concerning vehicle suspension and terrain profile.

- During modelling of vehicle suspension, the actual suspension elements characteristics and road wheels distribution has been introduced in order to attain an accurate demonstration of vehicle response.

- The simulating model excludes the assumption applied in the analytical investigation by a real representation of vehicle parameters.

- A parametric study concerning suspension parameters and road profile has been carried out using the proposed model in order to optimise and improve the vehicle dynamic performance.

Studying the possibility of integrating the active suspension with tracked vehicles is an effective issue in improving their dynamic performance.

\section{References}

[1] J.Yamakawa, K. Watanabe, A spatial motion analysis model of tracked vehicles with torsion bar type suspension, Department of Mechanical Engineering, The National Defense Academy, 1-10-20 Hashirimizu, Yokosuka 239-8686, Japan, 2004

[2] W. Schiehlen, B.Hu, Spectral Simulation and Shock Absorber Identification, International Journal of Non-Linear Mechanics 38, 2003, pp. 161-171

[3] S. Adhikari and J. Woodhouse, Identification of damping: Part 1, journal of sound and Vibration 2001, 243 (1), 43-61

[4] Stefaan W.R. Duym, Modeling and Identification for an Automotive Shock Absorber in the Context of Vehicle Dynamics, Vehicle System Dynamics, 33, 2000, pp. 261 285

[5] A.M Salem, T. Salahudien, Evaluation of Characteristics of Tracked Vehicles Torsion Bars, AMME-13 Conference May 27-29, 2008, MTC, Cairo, Egypt,

[6] Printed lectures of tanks department, Theory of Suspension, Chair of tanks, Military Technical College, Cairo, Egypt, 1974.

[7] A. M. Salem, W. Galal, Identification of Characteristics of Hydraulic Shock Absorbers Used in Light Weight Tracked Vehicles, ASAT-13 Conference May 2628, 2009, MTC, Cairo, Egypt,

[8] J Y Wong, J Preston-Thomas, Investigation into the effect of suspension characteristics and design parameters on the performance of tracked vehicles using an advanced computer simulation model, Proceedings 1988

[9] ANIL DHIR and SESHADRI SANKAR, Assessment of Tracked Vehicle Suspension System Using A Validated Computer Simulation Model, Journal of Terramechanics, Vol. 32, No. 3, pp. 127-149, 1995

[10] Corina Snadu, Jeffrey S. Freeman, Three- dimenstional Multibody Tracked Vehicle Modeling and simulation, ASME Design Engineering Technical Confereneces 2003, USA

[11] J.Y.Wong, John Wiley, Theory of Ground Vehicles, New York, Second edition, 1993 